\title{
Plasmid multimer status and not DNA topology likely affects luciferase assay reproducibility
}

\section{Michael G Jobling*,1 (iD}

${ }^{1}$ Retired faculty, Department of Immunology \& Microbiology, University of Colorado School of Medicine, Aurora, CO 80045, USA; ${ }^{2}$ uthor for correspondence: michael.jobling@ucdenver.edu

BioTechniques 69: 157-160 (September 2020) 10.2144/btn-2020-0009

First draft submitted: 17 January 2020; Accepted for publication: 6 May 2020; Published online: 12 June 2020

I was interested to see Tudini et al.'s letter 'Caution: Plasmid DNA topology affects luciferase assay reproducibility and outcomes' that, I believe, incorrectly identified plasmid supercoiling as a variable that affects reporter plasmid output [1]. It is important for all authors to consider issues that may affect experimental reproducibility, especially in a systemic manner. The authors set out to define the cause of considerable variability in a lux-based reporter transfection assay. They identified a problem (assay variability) and an unintended variable (reporter plasmid electrophoretic mobility), then stated that the mobility variance was consistent with differences in plasmid supercoiling. While they correctly pointed out that the topology of plasmid DNA can have an effect on its expression from reporter plasmids (their references 4-6) [2-4], I believe they were mistaken in assigning the variance in electrophoretic mobility to altered DNA topology (specifically, supercoiling or lack thereof) of the plasmid DNA.

They attested, without confirmation, that the electrophoretic mobility differences seen in individual replicate plasmid DNA preparations for several different reporter constructs represented supercoiled (SC, fast-migrating) or non-SC (NSC, slow-migrating) versions of each plasmid. All plasmid DNAs were reported as being purified using a commercial (Qiagen, MD, USA) miniprep kit but from an unidentified Escherichia coli host. The slow-migrating plasmid forms tended to produce lower relative luciferase activity upon transfection. The authors hypothesized a potential mechanism for reduced expression from their NSC plasmid DNA, namely that NSC DNA has a lower transfection 'rate' than SC DNA.

On the face of it, the hypothesis that variability in plasmid DNA topology is responsible for reduced expression of transfected reporter genes is plausible; however, several troubling points were not addressed by the study. No mechanisms were put forward, nor concerns raised, as to first, how different plasmid preparations of replicate clones might consist exclusively of either SC or NSC DNA; second, how the NSC conformation of slow-migrating DNA is 'often' retained after retransformation and subsequent repurification; or third, how restriction, ligation and retransformation of NSC plasmids was able to restore the SC condition in the majority of (but not all) cases. I am unaware of any mechanisms that could support a topological explanation for these observations.

I believe that the observed variances in electrophoretic mobility can be explained more easily and more completely not by topological differences in plasmids, but by the plasmid's multimeric status, with the major fast-migrating bands being monomeric SC and the major slow-migrating bands being dimeric SC forms of plasmid DNA. Close observation of the paper's Figure 1A shows that each plasmid preparation has a major faster mobility band and a minor slower migrating band; I believe these bands represent SC (or covalently closed circular [CCC]) and open circular (OC, or NSC, formed from CCC DNA by single-stranded nicking during the alkaline denaturation step, with subsequent relaxation of SC) forms of each plasmid, respectively. In recombination-proficient $E$. coli strains, plasmid multimers consisting of tandem repetitions of the monomeric form are often observed [5]. If a recA derivative of E. coli were used as the plasmid host (no genotype or strain name was given), as is often the case with commercially supplied cloning strains, then the multimeric state of any transformed plasmid would be maintained. Bacterial cells transformed with a dimeric plasmid will almost always produce CCC (SC) plasmid DNA preparations with reduced mobility compared with the monomeric form, even after retransformation. On the other hand, basic molecular biology tells us that retransformation of E. coli with NSC or OC DNA molecules should result in sealing of the nick and conversion to CCC/SC forms by cellular DNA ligase and topoisomerase. This would result in greater mobility of repurified plasmid DNA compared with original 'NSC' plasmid DNA; however, this was not seen. Additionally, complete digestion (with a single site cutter) of slower migrating (dimeric) plasmid molecules would generate linear monomers that, following self-ligation, would regenerate mostly faster migrating (monomeric) plasmid transformants, with occasional ligation of two monomer molecules or circularization of a partially digested dimeric fragment regenerating the slower migrating (dimeric) plasmids, fully explaining the data presented in Figure 2 of the paper.

The SC/NSC hypothesis could and should have been tested. Prolonged incubation of plasmid DNA at high $\left(65-70^{\circ} \mathrm{C}\right)$ temperatures (the conditions used to convert SC to NSC/OC [2-4]) should affect only the fast-migrating DNA (and convert it to the slow-migrating form); a shift to slower relative mobility of both forms of plasmid would indicate that both are, in fact, SC. Partial restriction digestion of both forms with a single cutter could test the monomer/dimer hypothesis, with the fast (monomeric) form only producing one linearsized band and the slower (dimeric) form generating an additional single-cut dimer band of twice the size.

It is also possible to envisage a mechanism whereby dimeric plasmid DNA molecules result in lower expression of reporter genes compared with monomeric forms, after transfection of equal amounts of DNA. For a given amount of DNA, the dimeric form has half 
as many molecules and may transfect half as many cells, but with a doubled gene dosage per cell. Dimer-transfected cells could show a slight reduction of overall transcription compared with monomers transfected into twice as many cells, because smaller monomers may be more efficiently trafficked to the nucleus for transcription [6].

In summary, the letter identified a potential source of sample variance in transfection assays, but incorrectly attributed the problem, without direct evidence, to differences in supercoiling; the data can be explained more readily by differences in the multimeric state of the reporter plasmids. This effect of the multimeric status of plasmid DNA on reporter plasmid assays apparently has not been reported previously. By not supporting their supercoiling theory with evidence or suggesting possible mechanisms that could produce differences in supercoiling, it is my belief that they reached an erroneous conclusion to explain their important novel observations.

\section{Financial \& competing interests disclosure}

The author has no relevant affiliations or financial involvement with any organization or entity with a financial interest in or financial conflict with the subject matter or materials discussed in the manuscript. This includes employment, consultancies, honoraria, stock ownership or options, expert testimony, grants or patents received or pending, or royalties.

No writing assistance was utilized in the production of this manuscript.

\section{Open access}

This work is licensed under the Attribution-NonCommercial-NoDerivatives 4.0 Unported License. To view a copy of this license, visit http: //creativecommons.org/licenses/by-nc-nd/4.0/

\section{References}

1. Tudini E, Burke LJ, Whiley PJ, Sevcik J, Spurdle AB, Brown MA. Caution: plasmid DNA topology affects luciferase assay reproducibility and outcomes. BioTechniques 67(3), 94-96 (2019).

2. Cherng JY, Schuurmans-Nieuwenbroek NME, Jiskoot W et al. Effect of DNA topology on the transfection efficiency of poly((2-dimethylamino)ethyl methacrylate)-plasmid complexes. J. Control. Release 60(2), 343-353 (1999).

3. Remaut K, Sanders NN, Fayazpour F, Demeester J, De Smedt SC. Influence of plasmid DNA topology on the transfection properties of DOTAP/DOPE lipoplexes. J. Control. Release 115(3), 335-343 (2006).

4. Weintraub H, Cheng PF, Conrad K. Expression of transfected DNA depends on DNA topology. Cell 46(1), 115-122 (1986).

5. Bedbrook JR, Ausubel FM. Recombination between bacterial plasmids leading to the formation of plasmid multimers. Cell 9(4, Part 2), 707-716 (1976).

6. Hornstein BD, Roman D, Arévalo-Soliz LM, Engevik MA, Zechiedrich L. Effects of circular DNA length on transfection efficiency by electroporation into HeLa cells. PLoS ONE 11(12), e0167537-e0167537 (2016) 